\title{
Pendeln zwischen zwei Oberzentren - von verlorener bis geschenkter Zeit
}

\author{
Martina Roggendorf ${ }^{1}$ and Claus-C. Wiegandt ${ }^{2}$ \\ ${ }^{1}$ infas Institut für angewandte Sozialwissenschaft GmbH, Friedrich-Wilhelm-Str. 18, \\ 53113 Bonn, Germany \\ ${ }^{2}$ Geographisches Institut der Universität Bonn, Meckenheimer Allee 166, \\ 53115 Bonn, Germany \\ Correspondence: Claus-C. Wiegandt (wiegandt@uni-bonn.de)
}

Received: 28 August 2017 - Revised: 12 December 2017 - Accepted: 22 February 2018 - Published: 16 March 2018

\begin{abstract}
Kurzfassung. The importance of commuting especially between large urban centers has become a major topic as a growing number of people cover increasing distances to reach their workplaces. Based on this approach, the article looks into commuting between the cities of Cologne and Bonn. Social ties to these centers were identified as a main cause for commuting. 20 interviews were analyzed to set up five social types of commuters who have very different perceptions of their daily commute: For the "stressed out" commuting means psychological distress, for the "unsatisfied" it is a disruptive factor while the "pragmatics" accept it as means to an end. The "opportunists" try to use the time of commuting in the most efficient way and for the "profiteers" it's even a valuable time-out in their daily routine. Besides aspects as duration and transportation mode, especially personal attitudes and qualities proved to be important.
\end{abstract}

\section{Einleitung: Zunehmendes Pendeln zwischen den Oberzentren}

„Stau, volle Bahnen und vertane Lebenszeit: Pendler brauchen gute Nerven“ (Tagesschau.de, 2017). So und vergleichbar titelten die Medien im April 2017 zur aktuellen Situation der Pendler in Deutschland, nachdem das Bundesinstitut für Bau-, Stadt- und Raumforschung (BBSR) eine Auswertung der Pendlerstatistik vorgelegt hatte (BBSR, 2017). Danach ist die Zahl der Pendler 2015 bundesweit auf einen neuen Rekordwert gestiegen: Mittlerweile arbeiten 60 Prozent aller Beschäftigten in einer anderen Gemeinde als sie wohnen im Jahr 2000 waren es mit $53 \%$ noch deutlich weniger. Aber nicht nur die Zahl der Pendler ist gestiegen, auch ihr Weg zum Arbeitsplatz ist von durchschnittlich 14,6 km im Jahr 2000 auf 16,8 km im Jahr 2015 angewachsen. Die zurückgelegten Kilometer im Berufsverkehr haben sich seit 1975 nahezu verdoppelt (BMVI, 2016).

Eine Besonderheit der vergangenen Jahre ist die steigende Anzahl der Pendler zwischen den Oberzentren. Mit Ausnahme von Hoyerswerda, Fürth und Schwabach gibt es heute in allen 127 Oberzentren Deutschlands einen positiven Pendler- saldo (BBSR, 2015). Die Oberzentren sind „trotz Abwanderung von Betrieben sowie der Suburbanisierung von Handel und Gewerbe weiterhin die wichtigsten Arbeitsplatzzentren“ (BBSR, 2015:7) geblieben. Seit einigen Jahren steigt zudem die Zahl derer, die zwar in einem Oberzentrum wohnen, jedoch in ein anderes Oberzentrum zum Arbeiten pendeln. 2002 waren es rund 800000 Personen, 2013 bereits über eine Million (BBSR, 2015). Die Verbindungen im motorisierten Individualverkehr sowie im öffentlichen Verkehr zwischen den großen Zentren sind in der Regel sehr gut und fördern diese Entwicklung. Insbesondere diejenigen Oberzentren, die räumlich am nächsten beieinander liegen, verzeichnen in der jüngsten Zeit die stärksten Zuwächse an Pendlerverflechtungen (BBSR, 2015:9).

Die Veröffentlichung der aktuellen Pendlerzahlen durch das BBSR hat zu einer hohen medialen Aufmerksamkeit geführt, die vor allem die negativen Folgen des Pendelns herausstellt. Im Tagesspiegel wird auf die ,fatalen Auswirkungen auf die Umwelt [...] und die Gesundheit der Betroffenen (Stress, Burn-out)“ (Bemmer, 2017) hingewiesen, in der Süddeutschen Zeitung heißt es dazu: „Die Zahl der Pendler 
ist so hoch wie nie. Das geht zulasten der Gesundheit, der Familien und der Umwelt.“ (Müller, 2017).

Auch in der wissenschaftlichen Debatte werden vor allem die gesellschaftlichen Herausforderungen thematisiert, die mit dem Pendeln verbunden sind. Neben der Zersiedlung und den Verkehrsproblemen in Folge der Suburbanisierung des Wohnens sind es die Umweltbelastungen und der Klimaschutz. Für den einzelnen Pendler werden vor allem die gesundheitlichen Folgen sowie die Auswirkungen auf die allgemeine Lebenszufriedenheit behandelt (Eckey et al. 2007; Kemen, 2016; Pfaff, 2012, 2014). Der Arbeitsweg wird beispielsweise als der Teil des Tages mit dem geringsten persönlichen Nutzen und der größten psychischen und physischen Belastung identifiziert (Rau, 2011; Kahneman et al., 2004).

Die Nutzung und Wahrnehmung des Arbeitsweges durch den Pendler selbst ist bislang jedoch im deutschsprachigen Kontext noch wenig erforscht (vgl. Poppitz, 2009). An dieser Stelle knüpft dieser Beitrag an Untersuchungen aus dem angelsächsischen Raum zur Nutzung von Reisezeiten an und will so eine Lücke in den Forschungen zum Berufspendeln schließen. Ziel des Beitrages ist es deshalb, den Arbeitsweg selbst in den Fokus des Erkenntnisinteresses zu stellen und zu klären, ob die Betroffenen selbst die negative Wahrnehmung des Pendelns teilen oder ob sie auch Vorteile am Pendeln erkennen.

Als Untersuchungsraum wurden die beiden Oberzentren Köln und Bonn gewählt, weil hier intensive Pendlerverflechtungen in beide Richtungen bestehen und aktuell starke $\mathrm{Zu}$ wächse zu verzeichnen sind. Nach einem Überblick zum Forschungsstand und zum Untersuchungsraum wird die methodische Herangehensweise vorgestellt. Die empirische Basis des Beitrags stellen problemzentrierte Leitfadeninterviews dar. Daraufhin erfolgt die Darstellung der Ergebnisse. Es wird dargelegt, warum die Berufstätigen pendeln und wie sie die Zeit auf dem Arbeitsweg nutzen. Schließlich werden fünf Pendlertypen zur unterschiedlichen Wahrnehmung des Arbeitsweges herausgearbeitet. Abschließend wird ein Fazit gezogen.

\section{Zum Stand der Forschung über die Rahmenbedingungen des Pendelns und die Nutzung des Arbeitsweges}

Wie eingangs erwähnt, ist die Zahl der Pendler, die sich immer wiederkehrend vom Wohnort zum Arbeitsort über Gemeindegrenzen hinweg bewegen, in Deutschland im 20. Jahrhundert kontinuierlich gestiegen (vgl. Ott und Gerlinger, 1992). Treibende Faktoren für das Pendeln waren der Ausbau des Verkehrsnetzes, der es den Beschäftigten ermöglicht, in immer kürzerer Zeit immer größere Distanzen zu überbrücken, sowie die zunehmende Motorisierung. Durch die Spezialisierung von Arbeitskräften sowie die Standortanforderungen und -entscheidungen der Unternehmen fand zudem eine immer größere Trennung der Funktionen „Wohnen“ und „Arbeiten“ statt (BBSR, 2015).

Die individuelle Entscheidung für tägliches Pendeln kann verschiedene Gründe haben. Einen wichtigen Grund stellt der Wechsel des Wohnstandortes aus privaten Motiven, wie etwa dem Wunsch nach einem eigenen Haus im Grünen oder auch dem Zusammenzug mit dem Partner bei Beibehaltung des Arbeitsplatzes, dar (Schneider et al., 2002a). Aber auch ein Arbeitsplatzwechsel kann bei Beibehaltung des Wohnortes zum Pendeln führen. Dem Wohnort wird in diesen Fällen eine höhere Bedeutung beigemessen als der Nähe zum Arbeitsplatz (Schneider et al., 2002a). Der Arbeitsplatzwechsel kann freiwillig erfolgen, etwa zugunsten eines höheren Verdienstes oder auch besserer Arbeitsbedingungen, aber auch unfreiwillig, etwa bei einer Versetzung oder einer Verlagerung der Arbeitsstelle. Als bedeutsame Einflussfaktoren gelten in diesem Zusammenhang Kinder, die bereits in Kindergarten oder Schule integriert sind, sowie ebenfalls berufstätige Partner (Poppitz, 2009).

Beeinflusst wird eine Entscheidung zum Pendeln in den letzten Jahrzehnten durch tiefgreifende Veränderungen der Arbeitswelt, die sich unter dem Begriff der „Entgrenzung“ zusammenfassen lassen (Gottschall und Voss, 2005; Läpple et. al., 2010; Pohl, 2009). Dazu gehören unter anderem die zeitliche Flexibilisierung der Arbeit (Kratzer und Sauter, 2005:112f.; Wörmer, 2016) und der zunehmende Anteil an Erwerbstätigen in sogenannten atypischen Beschäftigungsformen, zu denen befristet und geringfügig Beschäftigte, Teilzeitbeschäftigte sowie Zeitarbeitnehmer gehören (Kratzer und Sauter, 2005:89ff.). Damit entstehende Unsicherheiten führen zu einem Anstieg des berufsbedingten Pendelns, da ein Umzug aufgrund der ungewissen Zukunftsaussichten vermieden wird (Wörmer, 2016).

Ein bedeutender Einflussfaktor für die Entwicklung der beruflichen Mobilität ist die steigende Anzahl erwerbstätiger Frauen. Während 1970 nur etwa 46\% der Frauen berufstätig waren (Bundeszentrale für politische Bildung, 2010), sind es im Jahr 2015 bereits knapp 70\% (Statistisches Bundesamt, 2016). Infolgedessen kommt es zu einem Anstieg von Doppelverdienerhaushalten (Oostendorp, 2016). Im Gegensatz zur fordistischen Arbeitswelt, in der überwiegend der Mann als Hauptverdiener berufliche Mobilitätsentscheidungen traf, sind diese in der postfordistischen Arbeitswelt häufig das Ergebnis einer Abwägung der beruflichen Interessen beider Partner. In der Folge kommt es häufig zu längeren Pendelwegen (Oostendorp, 2016:181ff.).

Neben diesen makrostrukturellen Einflussfaktoren wirken sich auch verschiedene mikrostrukturelle Faktoren wie Alter, Geschlecht, Haushaltstyp und Bildungsgrad auf die Wahrscheinlichkeit für berufliche Mobilität aus (vgl. Kümmerling et al., 2008; Ruppenthal und Lück, 2009; Schneider et al., 2002b). Insbesondere junge Berufstätige weisen eine überdurchschnittliche berufliche Mobilität auf. Dies liegt zum einen daran, dass ältere Menschen oft durch die bereits vollzogene Familiengründung, Wohneigentum sowie die Integra- 
tion in soziale Netzwerke stärker an ihren Wohnstandort gebunden sind, und zum anderen daran, dass berufliche Neuorientierungen eher in frühen Phasen der Erwerbstätigkeit stattfinden (Ruppenthal und Lück, 2009). In Bezug auf das Bildungsniveau sind Erwerbstätige mit Hochschulabschluss deutlich mobiler als Erwerbstätige mit niedrigeren Bildungsabschlüssen. Gründe hierfür sind die höhere Spezialisierung sowie die stärkere Karriereorientierung. Aufstiegschancen werden auch dann wahrgenommen, wenn sie mit größeren räumlichen Veränderungen verbunden sind (Wörmer, 2016; Poppitz, 2009).

Neben diesen soziostrukturellen Merkmalen spielen auch individuelle Eigenschaften und Einstellungen eine maßgebliche Rolle für die Mobilitätsbereitschaft, wie „Heimatverbundenheit, Einstellungen zur Vereinbarkeit von Beruf und Familie und der subjektive Stellenwert von Partnerschaft, Kindern und Beruf" (Schneider et al., 2002b:76). Wenn Personen etwa ihren Wohnsitz an einem Ort behalten möchten, wird zwischen rationaler, sozialer und emotionaler Ortsbindung unterschieden (Reuber, 1993:6; Menzl, 2007:338ff).

In den letzten Jahrzehnten ist eine steigende Anzahl an Studien zu verzeichnen, die sich mit beruflicher Mobilität oder auch spezifischer mit dem Arbeitsweg und dessen Nutzung beschäftigen (vgl. Abraham und Schönholzer, 2012; Lyons und Urry, 2005; Pfaff, 2012; Poppitz, 2009; Rau, 2011; Wörmer, 2016). Dabei wurden bisher Themen wie der Zusammenhang zwischen der Verkehrsmittelnutzung auf dem Arbeitsweg und der Gesundheit (vgl. Kemen, 2016) oder aber der Zusammenhang zwischen der Pendelentfernung und der Lebenszufriedenheit (vgl. Pfaff, 2014) überwiegend mit quantitativen Methoden analysiert. Dies gilt weitgehend auch für Studien zu den Aktivitäten von Reisenden (vgl. Gamberini et al., 2013) auch unter Berücksichtigung der Digitalisierung (vgl. Camacho et al., 2017; Lyons et al. 2013; Wardman und Lyons, 2016). Einer der wenigen Aspekte, die qualitativ eingehender untersucht wurden, ist die Vereinbarkeit beruflicher Mobilitätsanforderungen mit familiären Lebensumständen (vgl. Jurczyk et al., 2009; Schneider et al., 2002a, b).

Der Arbeitsweg wirkt sich im Verhältnis zu anderen Aktivitäten überdurchschnittlich stark auf die körperliche sowie psychische Verfassung aus (vgl. Rau, 2011) und wurde von Kahneman et al. (2004) sogar als der Teil des Tages mit dem geringsten persönlichen Nutzen identifiziert. Auch Pfaff (2014) kommt zu dem Ergebnis, dass sich berufliches Pendeln negativ auf die Lebenszufriedenheit auswirkt. Ott und Gerlinger (1992:169ff.) benennen neun Belastungsfaktoren, die bei Pendlern zum Tragen kommen:

- vermindertes Zeitbudget,

- besondere Arbeitszeiten,

- verminderte soziale und familiäre Präsenz,

- Verkehrsstress und erhöhte Unfallrisiken,
- besondere Verkehrsunfallgefährdung,

- zusätzliche Mehrfachbelastungen,

- besondere Erkrankungsrisiken,

- erhöhte Arbeitsunfallgefährdung,

- sinkende Arbeitsmotivation.

Nach Redmond und Mokhtarian (2001) hat Pendeln jedoch nicht nur Nachteile: Zum einen kann die Zeit auf dem Arbeitsweg sinnvoll genutzt werden, beispielsweise zum Nachdenken, Entspannen, Telefonieren oder auch Lesen, zum anderen kann auch das „Reisen“ an sich eine positive Erfahrung sein (vgl. auch Jain und Lyons, 2008). Des Weiteren eröffnet der Arbeitsweg eine bessere gedankliche Trennung zwischen Wohn- und Arbeitsort: „Räumliche Distanz ermöglicht es, eine innerliche Distanz zwischen Arbeit und Privatleben aufzubauen." (Poppitz, 2009:292).

Ein relevanter Aspekt bei der Betrachtung des Arbeitsweges bezieht sich auf das genutzte Verkehrsmittel. Während bei ÖPNV- und MIV-Nutzern kein Zusammenhang zwischen der Länge des Arbeitsweges und der Gesundheit festgestellt werden konnte, zeigte sich bei Fahrradfahrern, dass mit der Länge des Arbeitsweges die Krankheitstage sowie der Body-Mass-Index sinken und das Wohlbefinden steigt (Kemen, 2016).

Die Gründe für die Wahl des Verkehrsmittels spielen in diesem Zusammenhang eine bedeutende Rolle. Nach Boltze et al. (2002) kann zwischen personen- und angebotsbezogenen Einflussgrößen unterschieden werden, die wiederum in objektive und subjektive Einflussgrößen differenziert werden können. Zu den personenbezogenen Einflussgrößen zählen Merkmale wie beispielsweise der Bildungsstand sowie der Wegezweck. Angebotsbezogene Einflussgrößen sind das jeweilige Verkehrsangebot, die Raumstruktur, die subjektive Wahrnehmung und Bewertung sowie die Informiertheit (vgl. Boltze et al., 2002).

Zudem wirkt sich auch die für den Weg benötigte Zeit auf die Verkehrsmittelwahl aus. Derzeit brauchen etwa $70 \%$ der Erwerbstätigen in Deutschland weniger als eine halbe Stunde für ihren Arbeitsweg, $23 \%$ sogar weniger als zehn Minuten. Zwischen 30 und 60 Minuten ist jeder vierte Pendler unterwegs und nur fünf Prozent benötigen mindestens eine Stunde. Die Fahrzeit variiert jedoch - die Erwerbstätigen aus den Ballungszentren sind länger unterwegs. Sie haben allerdings auch größere Strecken zurückzulegen (Statistisches Bundesamt, 2014).

\section{Zur Entwicklung der Pendlerverflechtungen zwischen Köln und Bonn}

Die Nutzung und Wahrnehmung des Arbeitsweges wird im Weiteren für die beiden Oberzentren Köln und Bonn untersucht. Die Millionenstadt Köln (1 061000 Einwohner, 2016) 


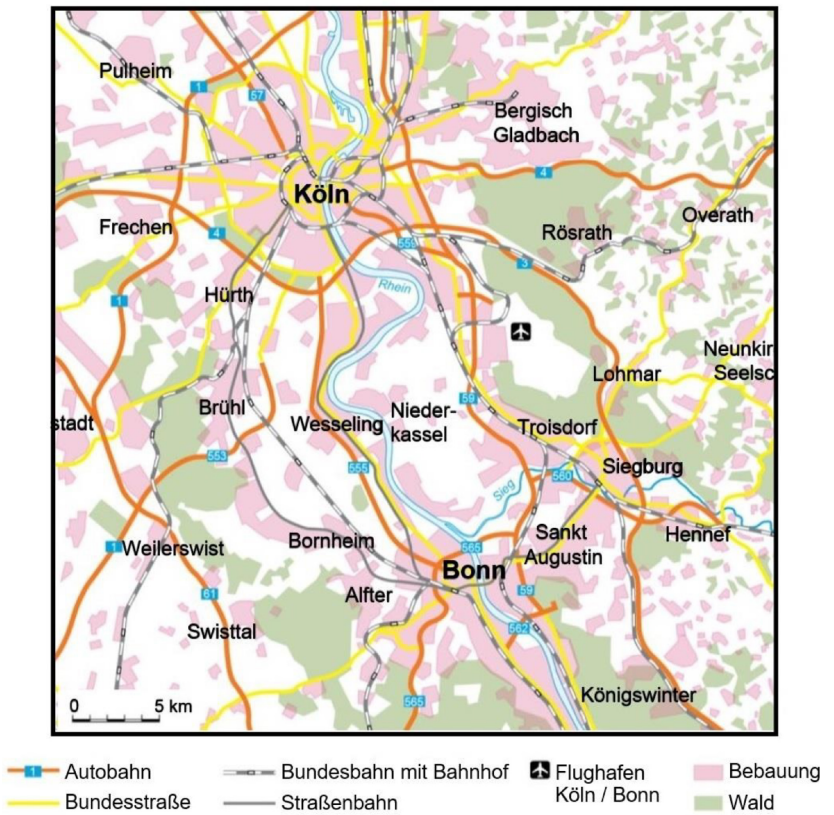

Abb. 1. Ausgewählte Verkehrsverbindungen zwischen Köln und Bonn (Quelle: Oostendorp 2016:61, verändert).

und die Bundesstadt Bonn (319000 Einwohner, 2016) sind auf beiden Seiten des Rheins verkehrstechnisch gut miteinander verbunden (s. Abb. 1). Zwei Autobahnen sowie verschiedene Bundesstraßen sichern eine gute gegenseitige Erreichbarkeit mit dem Auto. Zudem gibt es rechts- und linksrheinisch Bahnverbindungen mit einer Fahrzeit von durchschnittlich 20 bis 30 Minuten zwischen den verschiedenen Haltepunkten in Köln und Bonn. Ferner besteht die Möglichkeit, die Strecke mit den Straßenbahnlinien 16 oder 18 mit einer jedoch deutlich längeren Fahrzeit von etwa einer Stunde zurückzulegen.

Köln und Bonn gehören zu den Oberzentren in Deutschland mit den intensivsten Pendlerverflechtungen, die zudem die stärksten Zuwächse verzeichnen (BBSR, 2015). Täglich pendeln etwa 14000 Erwerbstätige zwischen den beiden Städten - sowohl von Köln nach Bonn als auch von Bonn nach Köln. Während bis 2007 die Anzahl der Einpendler nach Bonn aus Köln deutlich unter denen der Einpendler nach Köln aus Bonn lag, haben sich die Zahlen mittlerweile angeglichen (s. Abb. 2) (IT.NRW, 2017).

Die zunehmende Bedeutung der Pendlerverflechtungen wird auch an dem steigenden Anteil der Einpendler aus Köln bzw. Bonn an den gesamten Einpendlern nach Bonn bzw. Köln deutlich. Während 1998 etwa jeder fünfzehnte Berufstätige, der nach Bonn zum Arbeiten pendelte, aus Köln stammte, ist es heute bereits etwa jeder neunte. Für Köln zeigen sich ähnliche Entwicklungstendenzen, allerdings fällt die Steigerung etwas geringer aus. 1998 stammte mit einem Anteil von 3,49\% etwa jeder neunundzwanzigste Einpendler

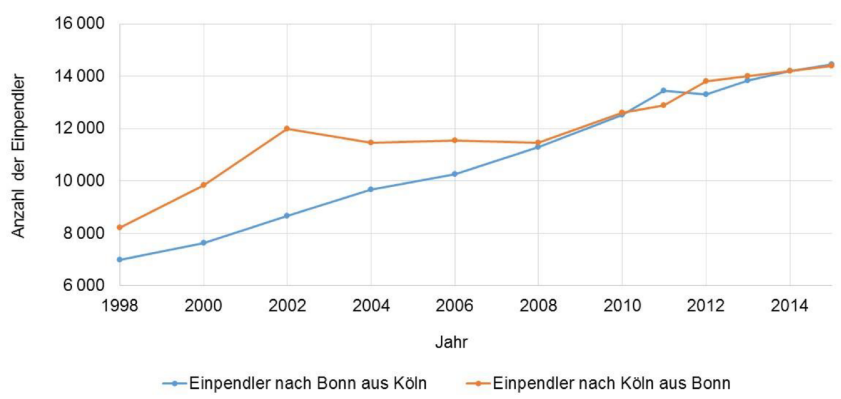

Abb. 2. Entwicklung der Einpendlerzahlen zwischen Köln und Bonn seit 1998 (Quelle: eigene Darstellung, Datenbasis: IT.NRW, 2017).

aus Bonn, aktuell ist es mit 4,55\% etwa jeder zweiundzwanzigste.

Auch die prozentualen Anteile der Einpendler an den Berufstätigen sind in diesem Kontext aufschlussreich: Insgesamt stammen 6,1\% aller Berufstätigen in Bonn aus Köln und $2 \%$ der Berufstätigen in Köln aus Bonn (eigene Berechnungen nach IT.NRW, 2017). Die unterschiedlichen Verhältnisse lassen sich mit der wesentlich größeren Anzahl an Erwerbstätigen in Köln erklären. Mit etwa 716000 Erwerbstätigen arbeiten hier mehr als dreimal so viele Menschen als in Bonn mit etwa 237000 (IT.NRW, 2017). In diesem Zusammenhang spielen auch die geringere Entfernung von Köln zu anderen Oberzentren sowie die bessere Anbindung von Köln an den Fernverkehr eine Rolle.

\section{Zur methodischen Herangehensweise}

Um die Nutzung und Wahrnehmung des Arbeitsweges zu untersuchen, wurde die Methode des problemzentrierten Leitfadeninterviews gewählt. Interviews gehören in der qualitativen Sozialforschung zu den am häufigsten eingesetzten Erhebungsinstrumenten (Flick, 2011).

Für die vorliegende Untersuchung wurden Anfang 2017 20 Berufstätige interviewt, die zum Zeitpunkt der Untersuchung zwischen Bonn und Köln bzw. in umgekehrter Richtung pendelten. Die Interviewpartner wurden mittels des theoretischen Samplings für die Untersuchung ausgewählt (vgl. Tab. 1). Entsprechend wurde darauf geachtet, dass sowohl Berufstätige befragt wurden, die in Köln wohnen und in Bonn arbeiten, als auch Berufstätige, die in Bonn wohnen und in Köln arbeiten. Zudem wurden sowohl Frauen als auch Männer sowie Teilzeit- und Vollzeitbeschäftigte befragt. Ein weiteres Kriterium für die Wahl der Interviewpartner war die Nutzung unterschiedlicher Verkehrsmittel - es wurden Nutzer des öffentlichen Personennahverkehrs, Autofahrer sowie ein Fahrradfahrer interviewt. Des Weiteren wurde beachtet, dass verschiedene Altersgruppen vertreten sind. Interessant wäre es gewesen, ergänzend Personen zu befragen, die das Pendeln aufgegeben haben. 
Tabelle 1. Interviewpartner.

\begin{tabular}{llllll}
\hline Name & $\begin{array}{l}\text { Wohn- } \\
\text { ort }\end{array}$ & & $\begin{array}{l}\text { Vollzeit/ } \\
\text { Teilzeit }\end{array}$ & $\begin{array}{l}\text { Dauer des } \\
\text { Arrangements }\end{array}$ & $\begin{array}{l}\text { Hauptsächlich } \\
\text { genutztes } \\
\text { Verkehrsmittel }\end{array}$ \\
\hline Herr O. & Köln & 29 & Vollzeit & 2 Jahre & Fahrrad \\
Frau N. & Köln & 28 & Vollzeit & 1 Jahr & ÖPNV \\
Herr M. & Köln & 58 & Vollzeit & 10 Jahre & Auto \\
Frau R. & Köln & 50 & Teilzeit & 10 Jahre & ÖPNV + Fahrrad \\
Frau J. & Köln & 44 & Vollzeit & 11 Jahre & ÖPNV + Fahrrad \\
Herr A. & Köln & 46 & Vollzeit & 4 Jahre & ÖPNV + Fahrrad \\
Frau P. & Köln & 52 & Teilzeit & 17 Jahre & Auto \\
Frau F. & Köln & 49 & Vollzeit & 2 Jahre & Auto \\
Herr G. & Köln & 47 & Vollzeit & 15 Jahre & Auto \\
Herr H. & Köln & 56 & Vollzeit & 5 Jahre & Auto \\
Herr S. & Köln & 44 & Vollzeit & 5 Jahre & Auto \\
Herr V. & Köln & 29 & Vollzeit & 7 Monate & Auto \\
Herr B. & Köln & 28 & Vollzeit & 2 Monate & ÖPNV + Fahrrad \\
Herr N. & Bonn & 36 & Vollzeit & 3 Jahre & ÖPNV \\
Frau K. & Bonn & 35 & Vollzeit & 6 Jahre & ÖPNV + Fahrrad \\
Herr K. & Bonn & 36 & Vollzeit & 11 Jahre & ÖPNV \\
Frau U. & Bonn & 58 & Vollzeit & 26 Jahre & ÖPNV \\
Frau B. & Bonn & 36 & Vollzeit & 9 Jahre & ÖPNV \\
Frau S. & Bonn & 33 & Teilzeit & 3 Jahre & ÖPNV \\
Frau H. & Bonn & 45 & Teilzeit & 3 Monate & Auto \\
\hline & & & & & \\
\hline
\end{tabular}

In den Interviews wurden Fragen zu den folgenden drei Themenblöcken gestellt: Wohnstandortwahl, Dauer und Nutzung des Arbeitsweges sowie Wahrnehmung und Erfahrungen. Anschließend wurden die Interviews in normales Schriftdeutsch transkribiert. Die Auswertung erfolgte in drei Phasen (Flick, 2011). Im ersten Schritt, der Einzelfallanalyse, wurden Kurzbeschreibungen der einzelnen Fälle erstellt, die sowohl wörtliche als auch sinngemäße Wiedergaben sowie eine erste Interpretation beinhalten. In der folgenden Fein- bzw. Tiefenanalyse wurde nach Sinnzusammenhängen in den Äußerungen gesucht und ein Kategoriensystem für den einzelnen Fall erstellt. Abschließend wurden in einem Fallvergleich Gemeinsamkeiten sowie Unterschiede der Fälle herausgearbeitet. Dabei wurden unterschiedliche Wahrnehmungen des Pendelns deutlich, die die Basis für die anschließend durchgeführte Typenbildung darstellten. Typisierungen gelten generell als bedeutsames, nicht quantifizierbares Erkenntnismittel in den Sozialwissenschaften (vgl. Mattissek et al., 2013), „,bei dem ein Objektbereich anhand eines oder mehrerer Merkmale in Gruppen bzw. Typen eingeteilt wird, sodass sich die Elemente innerhalb eines Typus möglichst ähnlich sind (interne Homogenität) und sich die Typen voneinander stark unterscheiden (externe Heterogenität)“ (Kluge, 1999:26f.).

Die Beschreibung des Arbeitsweges aus der Distanz könnte dazu geführt haben, dass er positiver beschrieben wurde als er tatsächlich wahrgenommen wird (vgl. Rau, 2011). In einer weiteren Untersuchung könnten deshalb die Empfindungen der Pendler auch in Form einer ethnographischen
Beobachtung unmittelbar während des Arbeitsweges erfasst werden. Dadurch könnten mögliche Differenzen noch genauer erfasst werden.

\section{Zu den Ortsbindungen als Begründung für das Pendeln}

Im Folgenden werden die empirischen Befunde der Untersuchung vorgestellt. Zunächst ist festzustellen, dass das berufliche Pendeln zwischen zwei großen Städten heutzutage auch in der Wahrnehmung der interviewten Pendler keine Ausnahme mehr darstellt. In Regionen wie Köln/Bonn scheinen durch die gute gegenseitige verkehrliche Erreichbarkeit räumliche Grenzen an Bedeutung zu verlieren:

„Wenn man sich anschaut, wie diese Städte miteinander verbunden sind, dann ist es tatsächlich so, dass wir ja im Prinzip von einem Agglomerationsraum sprechen können und das ja so ein bisschen eine künstliche Trennung ist.“ (Herr K.)

Auch wenn für die zu überbrückende Strecke täglich insgesamt zwei Stunden benötigt werden, gilt sie als ,,akzeptable Pendeldistanz". Für fast alle interviewten Pendler kommt deshalb ein Umzug nicht in Frage. In diesem Zusammenhang konnten in Anlehnung an Reuber (1993) drei unterschiedliche Faktoren identifiziert werden: die soziale, die emotionale sowie die rationale Ortsbindung.

Wie sich gezeigt hat, wird der Nähe zum sozialen Umfeld ein höherer Wert beigemessen als der Nähe zum Arbeits- 
platz, sodass ein Umzug die letzte mögliche Option darstellt. Es wird lieber zur Arbeit als zu den sozialen Kontakten gependelt. Diese Einstellung wird besonders am Beispiel von Herrn V. deutlich, der vor seinem Umzug nach Köln in Gehdistanz zu seinem Arbeitsplatz gewohnt hat und heute die einstündige Fahrt aufgrund seiner sozialen Kontakte in Kauf nimmt. Bei Pendlern, die Kinder haben, stellt zudem die soziale Bindung der Kinder einen wichtigen Einflussfaktor dar: „Also, bei der Frage, ob man nochmal umzieht, da spielen die schon eine große Rolle. Die Kinder sind jetzt natürlich hier verankert.“ (Frau B.). Lokale Sozialbeziehungen stellen - wie schon Wörmer (2016:209) festgestellt hat - ,im Kontext wiederkehrender Mobilitätsanforderungen den entscheidenden Parameter für ortsbezogene Bindungen und für eine zufriedenstellende alltägliche Lebensführung dar". Hierbei ist jedoch relevant, dass die Mehrheit der interviewten Pendler schon vor Beginn ihrer heutigen Beschäftigung in Köln bzw. Bonn an ihrem derzeitigen Wohnort ansässig waren. Eine starke lokale Verankerung durch soziale Netzwerke war dementsprechend meistens bereits vorhanden.

Neben der sozialen Ortsbindung konnte auch die emotionale Ortsbindung als relevanter Aspekt identifiziert werden - zumindest die Mehrheit der Pendler aus Köln betont die Identifikation mit ihrem Wohnort und ihrer Stadt ausdrücklich. Bei den interviewten Bonner Pendlern ist diese geringer ausgeprägt. Die rationale Ortsbindung spielt bei den Pendlern der beiden großen Oberzentren hingegen eine eher geringe Rolle. Lediglich von einigen Kölnern wird auf die bessere Verkehrsanbindung sowie das größere Freizeit- und Kulturangebot hingewiesen. Insgesamt kann festgehalten werden, dass die rationale Ortsbindung im Vergleich zur sozialen und emotionalen Ortsbindung für die interviewten Pendler nur eine untergeordnete Rolle spielt.

\section{Schlafen, lesen oder telefonieren? Die vielfältige Nutzung des Arbeitsweges}

Zwischen den beiden Oberzentren Bonn und Köln gibt es für die Pendler auf beiden Seiten des Rheins Möglichkeiten, ihren Arbeitsweg sowohl mit dem Auto als auch mit dem ÖPNV zurückzulegen (vgl. Abschnitt 4). Die Gründe für die Verkehrsmittelwahl sind vielfältig. Ausschlaggebend für die Entscheidung, den Arbeitsweg mit dem Auto zurückzulegen, ist die Fahrzeit. Vor allem Pendler, die im ÖPNV mehrfach umsteigen müssen, um zu ihrer Arbeitsstelle zu gelangen, ziehen das Auto vor. Für Pendler, die beruflich viele auswärtige Termine wahrnehmen müssen, sowie familiär eingebundene Pendler stellt zudem die Flexibilität, die das Auto bietet, ein wichtiges Entscheidungskriterium dar.

Bei der Begründung für das Zurücklegen des Arbeitsweges mit dem ÖPNV werden sowohl personenbezogene als auch angebotsbezogene Einflussfaktoren angeführt. Einige der interviewten Pendler gaben an, dass sie kein Auto besitzen und auch kein Bedürfnis haben, sich dieses anzuschaffen.
Wie die MIV-Nutzer benennen auch die ÖPNV-Nutzer die Fahrzeit als Einflussfaktor. In diesem Zusammenhang spielt auch die Unberechenbarkeit der Autofahrt für die interviewten ÖPNV-Nutzer eine Rolle. Zudem wird das Fahren im ÖPNV als ,weniger stressig“ bzw. ,entspannter“ empfunden und die Zeit als sinnvoll nutzbar bewertet, ,,während man halt beim Autofahren nichts anderes tun würde als Autofahren“ (Frau K).

Pendler, die mit dem Auto zur Arbeit fahren, sind im Gegensatz zu Pendlern, die ihren Arbeitsweg mit dem ÖPNV zurücklegen, räumlich von den anderen Pendlern getrennt. Sie fahren ihre Strecke in der Regel alleine. Dadurch können sie relativ ungestört ihrer jeweils präferierten Tätigkeit nachgehen, wobei sie allerdings durch das Führen des Fahrzeugs stark eingeschränkt sind. Die am häufigsten genannte Tätigkeit im Auto ist das Radiohören bzw. alternativ auch das Hören von Musik mittels CD oder Handy. Für eine Befragte ersetzt das morgendliche Radioprogramm das Zeitungslesen. Zum Telefonieren nutzen nicht alle befragten Autofahrer die Zeit im Auto. Dies wird zum einen mit einer fehlenden Freisprechanlage begründet, zum anderen damit, dass beim Telefonieren nicht mehr die notwendige Konzentration auf den Verkehr gewährleistet werden kann. Pendler, die hingegen regelmäßig auf ihrem Arbeitsweg telefonieren, führen zum einen private Gespräche, zu denen sie sonst nicht kommen, zum anderen aber auch berufliche Telefonate. Des Weiteren wird der Arbeitsweg zum Nachdenken sowie zum Trinken des morgendlichen Kaffees genutzt. Ein Interviewter berichtet beispielsweise, dass er, um morgens Zeit zu sparen, das Trinken des Kaffees von seiner Küche in sein Auto verlagert hat.

Im ÖPNV sind die Nutzungsmöglichkeiten hingegen vielfältiger als im Auto, da sich die ÖPNV-Nutzer während ihrer Fahrt nicht auf die Steuerung des Verkehrsmittels konzentrieren müssen. Im Gegensatz zum Auto sind die Nutzer des ÖPNVs jedoch räumlich nicht von anderen Pendlern getrennt. Dies hat einen entscheidenden Einfluss auf die Zeitnutzung. Die wenigsten der interviewten ÖPNV-Fahrer nutzen den Arbeitsweg für berufliche Zwecke. Hauptsächlich wird privaten Tätigkeiten nachgegangen. Mögliche Gründe hierfür sind die vergleichsweise kurze Bahnfahrt sowie die im Vergleich zu Fernverkehrszügen schlechtere Ausstattung der Nahverkehrszüge. Ein Gesprächspartner, der in seiner Studentenzeit die Zugfahrten bewusst zur Vorbereitung auf Seminare genutzt hat, erklärt seine Entscheidung, den Arbeitsweg nur noch für private Zwecke zu nutzen, folgendermaßen: ,Jetzt ist das so, dass ich mir denke, ,Ich arbeite halt irgendwie acht Stunden. Da muss ich jetzt im Zug nicht auch noch arbeiten'."(Herr B.).

Bei den ÖPNV-Nutzern spielt die Umgebung im Zug eine wichtige Rolle. Zum einen kann sie eher als störend wahrgenommen werden, sodass sich die Pendler mit Hilfe elektronischer Geräte bewusst abschotten, zum anderen kann sie jedoch auch der eigenen Unterhaltung dienen, indem beispiels- 
weise Gesprächen zugehört wird oder Textnachrichten des Sitznachbarn mitgelesen werden.

Die meisten ÖPNV-Nutzer lesen auf ihrem Arbeitsweg jedoch ihre eigens mitgebrachte Lektüre entweder in Form der klassischen Printmedien oder inzwischen auch digital. Während sich manche ÖPNV-Nutzer mittels „Zeitungs-Apps“ oder auch der entsprechenden Printausgaben über die aktuellsten Entwicklungen informieren, bevorzugen andere eher „leichte Lektüre“. Auch wenn die Pendler keinen Sitzplatz bekommen, versuchen einige von ihnen, die Zeit im Zug zum Lesen zu nutzen: ,Also, wenn es ganz eng ist, dann geht es nicht, aber wenn man ein bisschen Platz hat zum Stehen, dann kann man sich mit einer Hand beim Lesen festhalten.“ (Frau B.).

Andere ÖPNV-Nutzer geben an, dass sie während der Zugfahrt selten lesen und stattdessen ,eher die modernen Medien“ nutzen, um Podcasts oder Musik zu hören. Dies wird zum einen als Mittel zur Abschottung gegen die Umgebungsgeräusche genutzt, fördert zum anderen jedoch auch die Entspannung. Einige der Interviewten nutzen diese Methode der Abschottung nur, wenn in ihrer direkten Nähe sehr laute Unterhaltungen geführt werden. Die meisten ÖPNVNutzer empfinden die Atmosphäre im Zug insbesondere morgens allerdings als wenig störend. Ein Interviewter betont, dass in den Zügen in der Regel eine sehr gedämpfte Stimmung herrscht, da ,die Berufspendler ja alle dasselbe Schicksal haben." (Herr A.).

Während sich manche Pendler also gezielt von der Außenwelt abgrenzen, nutzen andere ihre Umgebung zur eigenen Unterhaltung. So berichtet eine Pendlerin, dass sie es sehr interessant findet, „,mal zu gucken, was die Leute so machen“ und während des Musikhörens häufig mitliest, was ihre Sitznachbarn „so auf ihren Handys tippen“ (Frau N.). Eine andere Pendlerin hört anderen Bahnfahrern bewusst bei ihren Unterhaltungen zu: ,Das ist auch immer sehr interessant. Also, da muss man dann gar nicht lesen oder so. Man tut halt so, als würde man lesen, aber man hört einfach den Gesprächen zu. Das ist auch immer sehr lustig.“ (Frau S.).

Die Mehrheit der interviewten Pendler bevorzugt es, sich im Zug nicht zu unterhalten und in Ruhe zu lesen, Musik zu hören oder anderen Tätigkeiten nachzugehen. Dementsprechend fährt der überwiegende Teil der Pendler lieber alleine Zug. Vor allem morgens werden in der Regel keine Verabredungen getroffen, abends wird der Weg nach Hause hingegen zum Teil mit Kollegen zurückgelegt.

Einige Pendler präferieren es jedoch, sich während der Zugfahrt zu unterhalten. Eine Gesprächspartnerin begründet dies damit, dass sie auch generell ,eher der kommunikative Typ ist, der dann doch mal jemanden sucht, den er kennt" (Frau S.). Die Rückfahrt mit den Kollegen kann sich auch zu einem sozialen Ereignis entwickeln, wie eine andere Gesprächspartnerin berichtet. Als noch zwei andere Kollegen mit ihr zwischen Köln und Bonn gependelt sind, wurde jeweils zum Wochenende das ,Freitags-Nachmittags-
Feierabend-Wochenende-Bier“" im Zug getrunken, was sie als ein schönes Ritual empfunden hat.

Eine weitere recht häufig genannte Beschäftigung im Zug ist das Schreiben von WhatsApp-Nachrichten oder E-Mails. Änderungen haben sich aus den zum Lesen verwendeten Medien ergeben. So berichten Pendler, dass sie früher noch die Printversion der Tageszeitung gelesen haben, während sie mittlerweile das Smartphone oder das iPad hierfür verwenden. Vereinzelt wird die Bahnfahrt auch genutzt, um Handyspiele zu spielen, aber auch um zu frühstücken, Abendbrot zu essen, Sudokus zu machen oder zu schlafen.

Sowohl bei den Autofahrern als auch den Bahnfahrern zeigen sich Unterschiede in der Nutzung der Zeit zwischen dem Hin- und dem Rückweg: Der Hinweg wird eher genutzt, um langsam wach zu werden und sich auf den Tag vorzubereiten, der Rückweg eher zum Einstimmen auf die anstehende Freizeit. Dies zeigt sich auch in den jeweils ausgeübten Tätigkeiten. So liest eine interviewte Pendlerin beispielsweise morgens Tageszeitung, da dies für ihren Job wichtig ist, und abends ein Buch zur privaten Unterhaltung.

Insbesondere die Nutzung des ÖPNV eröffnet also viele verschiedene Möglichkeiten, die Zeit auf dem Arbeitsweg zu nutzen. Dabei wird meist privaten Tätigkeiten nachgegangen, die der Erholung oder Unterhaltung dienen. Die Nutzung des Arbeitsweges kann dabei dessen Wahrnehmung so positiv beeinflussen, dass einige Pendler mitunter von dieser Zeit profitieren, anstatt sie als verloren zu betrachten (vgl. Lyons und Urry, 2005). Für die Zeit des Pendelns haben sich also individuell Routinen eingestellt, die helfen, sich mit dem Pendeln abzufinden.

\section{Von verlorener bis geschenkter Zeit - fünf Pendlertypen zur Wahrnehmung des Arbeitsweges}

Für die Wahrnehmung des Arbeitsweges erfolgt eine Typisierung der interviewten Pendler entlang der beiden Merkmale ,Wahrnehmung der für den Arbeitsweg aufgewendeten Zeit" und „Bedeutung des Arbeitsweges“. Es konnten fünf Pendlertypen gebildet werden: die „Gestressten“, die „Unzufriedenen“, die „Pragmatiker“, die „Opportunisten“ und die „Profiteure“ (s. Abb. 3). Die Übergänge zwischen den fünf Typen sind jedoch fließend.

Sowohl die Gestressten, die Unzufriedenen als auch die Pragmatiker nehmen die für den Arbeitsweg aufgewendete Zeit eher oder gänzlich negativ wahr. Während die Pragmatiker den Arbeitsweg als Mittel zum Zweck bewerten, stellt dieser für die Unzufriedenen einen Störfaktor und für die Gestressten sogar eine große psychische Belastung dar. Die Opportunisten und Profiteure stehen dem Zeitfaktor hingegen neutral oder sogar positiv gegenüber und unterscheiden sich dahingehend voneinander, dass die Opportunisten lediglich das zeitliche Potenzial des Arbeitsweges erkennen und ihn bestmöglich gestalten, sodass er für sie einen Nutzen hat, 


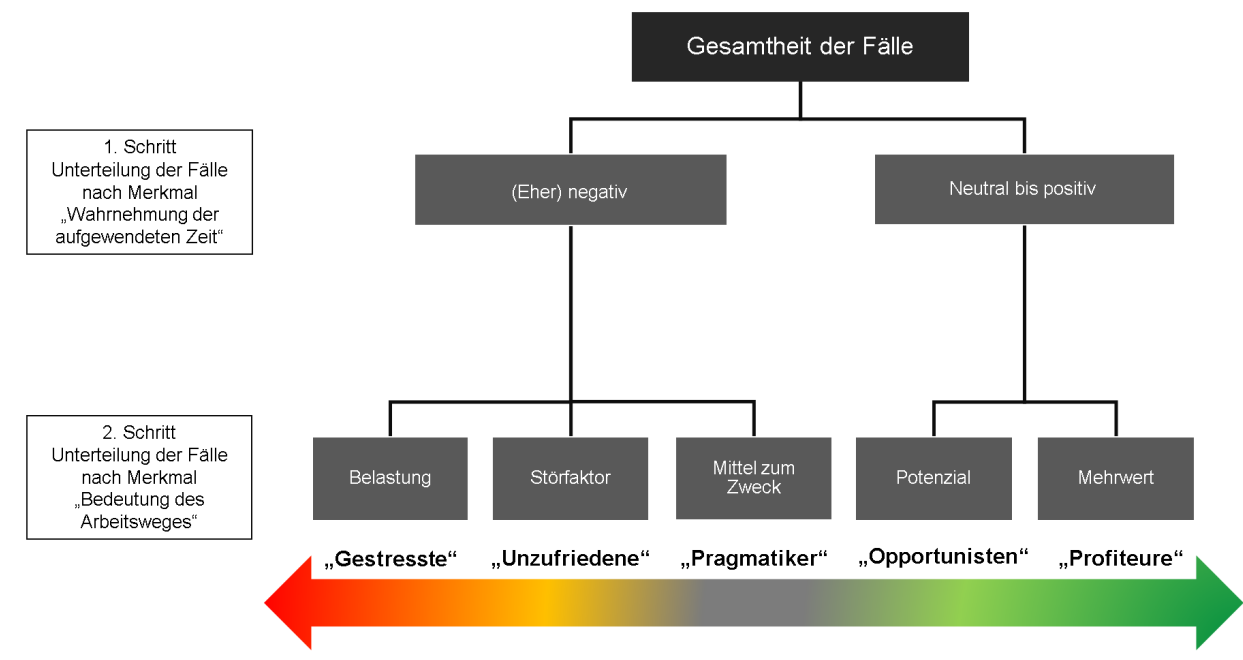

Abb. 3. Fünf Pendlertypen (Quelle: eigene Darstellung).

wohingegen die Profiteure den Arbeitsweg an sich als Mehrwert in ihrem Tagesablauf ansehen.

\subsection{Die „Gestressten“ - Pendeln als psychische Belastung}

Der Typ „Gestresste“ umfasst Pendler, die den Arbeitsweg als starke zeitliche sowie psychische Belastung wahrnehmen. Die Zeit, die auf dem Arbeitsweg verbracht wird, wird eher oder gänzlich zur Arbeitszeit gerechnet und als ,verlorene Zeit" angesehen. Im Gegensatz zur bezahlten Arbeitszeit bringt ihnen die Zeit des Pendelns keinen Nutzen:

„So gesehen habe ich pro Woche 40 Stunden Arbeit, für die ich bezahlt werde und zehn Stunden Pendelei beziehungsweise Arbeitszeit, für die ich nicht bezahlt werde." (Herr N.)

Für die „Gestressten“ ist der Arbeitsweg jedoch nicht nur eine zeitliche Belastung, sondern in Abgrenzung zu dem Typ der „Unzufriedenen“ auch ein Stressfaktor und wirkt sich folglich deutlich stärker auf ihre psychische Verfassung aus. Die Gründe dafür sind unterschiedlich und unter anderem vom genutzten Verkehrsmittel abhängig. Im ÖPNV werden vor allem mitfahrende Personen als Stressfaktor wahrgenommen. Bei Autofahrern sind einerseits die anderen Verkehrsteilnehmer ein Anlass, sich aufzuregen, andererseits stellt auch der morgendliche Zeitdruck einen Stressfaktor dar, denn wer nicht früh genug losfährt, gerät in den Hauptberufsverkehr.

Doch nicht nur der tatsächliche Weg kann mit Stress verbunden sein, sondern bereits der Gedanke an den bevorstehenden Weg kann das Wohlbefinden beeinträchtigen: „Manchmal hat man natürlich so ein bisschen das Grauen und denkt sich ,Morgen wieder. Ich habe keinen Bock, morgen wieder im Stau zu stehen'.“ (Herr V.).
Eine Gemeinsamkeit und mögliche Ursache für die insgesamt sehr negative Wahrnehmung der „Gestressten“ stellt der Umstand dar, dass der Weg zur Arbeit vor diesem Arrangement wesentlich kürzer war und erst seit vergleichsweise geringer Zeit zwischen Bonn und Köln gependelt wird. Trotz der großen psychischen Belastung plant allerdings nur ein Gesprächspartner, etwas an der Situation ändern zu wollen, und berichtet, dass er aktiv nach einer neuen Stelle an seinem jetzigen Wohnort sucht.

\subsection{Die „Unzufriedenen“ - Störfaktor Arbeitsweg}

Die Pendler, die den Typ „Unzufriedene“ bilden, bewerten genau wie die „Gestressten“ die Zeit für den Arbeitsweg als „,verlorene“ oder auch „verschenkte Zeit“. Mit dem Arbeitsweg als „Zeitfresser“ verbinden sie keinen persönlichen Nutzen. Im Unterschied zu den „Gestressten“ stellt der Arbeitsweg für sie zwar keinen Stressfaktor, aber einen Störfaktor dar.

Der Arbeitsweg wird von den „Unzufriedenen“ aufgrund verschiedener Faktoren als „lästig“ empfunden. Bei der Nutzung des ÖPNV entsteht das Gefühl, ,unfrei“ zu sein. Dies steht für eine Gesprächspartnerin in Verbindung mit einer von ihr wahrgenommenen aggressiven Stimmung in den öffentlichen Verkehrsmitteln:

„Wenn man einsteigt und die Leute schubsen und drängeln und schieben einen rücksichtslos da rein und können nicht warten, bis alle anderen ausgestiegen sind. Das finde ich wirklich ganz, ganz schlimm und das nervt mich auch jedes Mal tierisch. Da kriege ich dann richtig schlechte Laune.“ (Frau N.)

Obwohl der Arbeitsweg von allen Unzufriedenen als ,lästig" wahrgenommen wird, stellt ein Umzug für sie jedoch 
keine Option dar. Die soziale Bindung an Köln bzw. Bonn durch die Familie, den Partner und die Freunde wird dabei als bedeutendster Einflussfaktor genannt.

\subsection{Die „Pragmatiker“ - Mittel zum Zweck}

Zur Gruppe der „Pragmatiker“ gehören Pendler, die den Arbeitsweg zwar als sehr zeitintensiv oder zeitraubend beschreiben, diesen jedoch als notwendiges „Mittel zum Zweck“ akzeptieren. Generell würden die meisten „Pragmatiker" zwar gerne auf den langen Arbeitsweg verzichten, doch er stellt für sie keinen bedeutenden Stör- oder Stressfaktor dar: „Der Arbeitsweg muss halt gemacht werden. Es ist halt so", sagt Herr S. dazu. Ein Grund für diese Einstellung kann der jeweilige Job sein, wie ein anderer Interviewpartner betont: „Der Job hier ist es mir wert, dass ich diese Zeit halt verbringen muss." (Herr A.).

Die pragmatische Einstellung ist jedoch nicht notwendigerweise von vorneherein gegeben, sondern kann sich auch erst mit der Zeit einstellen. Ein Gesprächspartner, der seit fünf Jahren pendelt, erzählt, dass er im Laufe der Zeit eine „gewisse Gelassenheit“ gelernt hat. Ein anderer Gesprächspartner, der seit vier Jahren pendelt, betont, dass es sich dabei um ,eine Gewöhnungssache“ handelt und ihm das Pendeln mit der Zeit leichter gefallen ist.

Die „Pragmatiker“ äußern sich kaum negativ über ihren Arbeitsweg. Insbesondere die Stimmung in den Pendelzügen wird von ihnen als in der Regel angenehm beschrieben: „Ich empfinde beim Zugfahren so eine Ruhe im Zug, die irgendwie eingehalten wird. So kommt es mir jedenfalls vor und das mag ich eigentlich morgens auch.“ (Herr S.). Ein Grund für diese Stimmung wird in der Zusammensetzung der $\mathrm{Zu}$ greisenden gesehen:

„In dem Fall, zwischen Köln und Bonn, sind es halt sehr viele Studenten, die unterwegs sind. In den späten Zügen eher Studenten und in den frühen Zügen eher die arbeitende Bevölkerung. Und, wie gesagt, dann ist man mit routinierten Pendlerprofis quasi unterwegs und alle verhalten sich gleich. Nach einem Muster. Und da gibt es wenig Störendes finde ich." (Herr A.)

Da sich die „Pragmatiker“ mit dem Pendeln arrangiert haben, stellt sich für sie die Frage nach einem Umzug nicht.

\subsection{Die „Opportunisten“- Das Beste daraus machen}

Die „Opportunisten“ bewerten die für den Arbeitsweg benötigte Zeit neutral bis positiv und empfinden durch die bewusste Nutzung dieser Zeit einen Mehrwert. Diese Einstellung kann sich allerdings erst im Laufe der Zeit entwickeln:

„Also, ich würde nicht mehr sagen, dass ich das jetzt als weggeworfene Zeit hinnehme. Sonst würde ich was ändern müssen. Wenn ich zwei Stunden am Tag für die kurze Strecke im Auto sitze, sagt der gesunde Menschenverstand, dass das Blödsinn ist und ich das ändern sollte. Aber da sehe ich im Moment wenig Optionen und deswegen sage ich mir ,Dann mach was draus‘. Insofern hat sich doch schon was geändert, aber erst seit anderthalb Jahren würde ich sagen." (Herr M.)

Die „Opportunisten“ sehen das zeitliche Potenzial des Arbeitswegs und machen das Beste daraus. Sie sorgen somit selbst dafür, dass der Arbeitsweg für sie einen Mehrwert hat. Dieser Mehrwert kann verschiedene Aspekte umfassen. Ein Gesprächspartner nutzt die Zeit im Auto beispielsweise ,bewusst aus“, indem er Musik oder auch Hörbücher hört und über Dinge nachdenkt, zu denen er zuhause nicht in Ruhe kommt. Sein Auto bezeichnet er deshalb als eine Art ,rollendes Wohnzimmer“. Ein anderer Gesprächspartner argumentiert, dass es für ihn letztlich keinen Unterschied macht, ob er zuhause auf der Couch sitzt und liest oder Musik hört oder dies im Zug tut. Des Weiteren werden besondere Erlebnisse während des Pendelns positiv herausgestellt. So berichtet ein Gesprächspartner etwa von Sonnenaufgängen, die er während seines Arbeitsweges beobachten konnte: „Das ist einfach bereichernd, weil ich das wahrscheinlich gar nicht sehen würde, wenn ich nicht pendeln würde." (Herr B.).

Oft haben die „Opportunisten“ ihr Verhalten infolge von negativen Erfahrungen geändert und sich Strategien angeeignet, um das Pendeln für sich selber angenehmer zu gestalten:

„Weil mich dieses nervige Im-Stau-stehen, das Suchen nach alternativen Routen und das Anbrüllen des Vordermanns, der nicht fährt oder jemanden reinlässt, irgendwo kaputt gemacht hat. Das hat mich so derart genervt, weil mein Ziel dadurch behindert wird. Und jetzt sage ich mir ,Okay, ich nehme das so, wie es ist' " (Herr M.)

ÖPNV-Fahrer meiden in diesem Kontext beispielsweise unzuverlässige Bus- oder Straßenbahnlinien. Einen Umzug zieht keiner der ,, Opportunisten“ aktuell in Betracht.

\subsection{Die „Profiteure“ - Zeit zum Entspannen}

Die Gruppe der „Profiteure“ bilden Pendler, die ihren Arbeitsweg als wertvollen Bestandteil ihres Alltags wahrnehmen. Dementsprechend wird die dafür benötigte Zeit positiv bewertet. Die auf dem Arbeitsweg verbrachte Zeit wird beispielsweise als „Entspannungszeit“ oder sogar als „geschenkte Zeit" bezeichnet.

Das Pendeln führt für die „Profiteure“ zu einer klaren räumlichen und somit gefühlten Trennung zwischen Privatund Arbeitswelt. Die positive Wahrnehmung spiegelt sich auch darin wider, wie die „Profiteure“ ihre Umgebung beim Pendeln wahrnehmen und inwiefern sie sich davon beeinflussen lassen. Die ÖPNV-Nutzer nehmen in den Zügen eine spezifische Atmosphäre wahr, die ein Interviewter als „Gentlemen's Agreement“" zwischen den Pendlern bezeichnet. Dies 
beruht seiner Meinung nach auf der unausgesprochenen Regel, ,dass man sich seinen Platz nimmt und alle anderen in Ruhe lässt." (Herr K.). Begründet wird dies von einer anderen Pendlerin mit dem homogenen sozialen Status der Pendler auf der Strecke zwischen Bonn und Köln:

„Das sind eben viele Leute, die berufstätig sind [...], die einfach nur zur Arbeit und abends wieder nach Hause wollen. Viele arbeiten an der Uni. Das merkt man eben auch. Also, es ist sicherlich ein Background da, der relativ homogen ist. [...] Und dadurch entsteht eben auch eine andere Atmosphäre. Das ist sehr kollegial.“ (Frau U.)

Die besondere Zusammensetzung der Pendler im Zug zeigt sich vor allem bei Störungen im Betriebsablauf. Mit solchen Situationen wird nach Aussage einer Interviewpartnerin eher komödiantisch umgegangen und es entsteht ,eine sehr nette Kommunikation“ über das gemeinsame Schicksal. Ebenso wird vom Kennenlernen neuer Leute berichtet oder einem Zugfahrer, der sich mit den Fahrgästen über die Lautsprecher unterhalten hat. Eine mögliche Ursache für die positive Wahrnehmung der , Profiteure“ stellt die vergleichsweise lange Zeit dar, in der sie bereits zwischen Köln und Bonn pendeln - diese liegt zwischen fünf und 16 Jahren. Die „Profiteure" sind mit dem aktuellen Arrangement zufrieden und verspüren dementsprechend keinen Wunsch umzuziehen.

\section{Fazit}

Ziel des Beitrages war es, am Beispiel von Berufspendlern zwischen den beiden Oberzentren Köln und Bonn zu untersuchen, wie der tägliche Arbeitsweg genutzt und wahrgenommen wird. Anlass für die Untersuchung waren zum einen die wachsenden Pendlerzahlen in Deutschland, zum anderen die einseitige Berichterstattung über die physischen und psychischen Belastungen des Pendelns. Von einem ,,massenwirksamen Elend“ einer ,Völkerwanderung der Zivilgesellschaft" ist hier im Feuilleton der Süddeutschen Zeitung gar die Rede (Matzig, 2017).

Im Ergebnis zeigt sich, dass die interviewten Pendler die Strecke zwischen Bonn und Köln als eine annehmbare Pendeldistanz erachten. Es kommt zwar zu ganz unterschiedlichen Wahrnehmungen und individuellen Anpassungsstrategien bei den Pendlern, ein Umzug kommt für die Interviewten in der Regel jedoch nicht in Frage. Ursächlich hierfür ist vor allem die soziale Ortsbindung durch den Partner, die Familie, die Freunde, aber auch die Nachbarschaft. Für die Kölner spielt zudem die emotionale Ortsbindung eine Rolle. Die rationale Ortsbindung hat sich als vergleichsweise unbedeutend herausgestellt. Die gesamtgesellschaftlichen Probleme, die das Pendeln auslöst, wurden in den Interviews nicht thematisiert.

Eine wesentliche Erkenntnis der Studie ist, dass die Bedeutungszuschreibung durch die interviewten Pendler sehr unterschiedlich ausfällt. Es konnten fünf Pendlertypen anhand der Merkmale ,Wahrnehmung der aufgewendeten Zeit“ und „Bedeutung des Arbeitsweges“ identifiziert werden: Die „Gestressten“, für die der Arbeitsweg eine große psychische Belastung darstellt, die „Unzufriedenen“, die den Arbeitsweg als Störfaktor wahrnehmen, die „Pragmatiker“, die den Arbeitsweg als notwendiges Mittel zum Zweck akzeptieren, die „Opportunisten“, die die Zeit für den Arbeitsweg bestmöglich nutzen und die „Profiteure“, für die das Pendeln eine wertvolle „Auszeit“" von ihrem Alltag darstellt. Zusammenfassend kann festgehalten werden, dass die Gestressten den Arbeitsweg am negativsten und die Profiteure ihn am positivsten wahrnehmen. Die Unzufriedenen, die Pragmatiker sowie die Opportunisten können dazwischen eingeordnet werden.

Die Gründe für diese unterschiedlichen Bedeutungszuschreibungen sind äußert komplex. Hierzu gehören neben der Dauer des Arrangements, der zeitlichen Beanspruchung und Häufigkeit des Pendelns, dem genutzten Verkehrsmittel und der Zufriedenheit mit dem aktuellen Arbeitsplatz auch die auf dem Arbeitsweg gemachten Erfahrungen. Als bedeutsam haben sich zudem vor allem persönliche Einstellungen und Eigenschaften, wie beispielsweise die Fähigkeit, seine Umgebung auszublenden, herausgestellt.

Der vorliegende Beitrag ergänzt die vorwiegend quantitativen Ansätze zur Wahrnehmung des Pendelns (vgl. Pfaff, 2014) um eine qualitative Dimension. Die Ergebnisse schlieBen unter anderem an die Studien von Jain und Lyons (2008), Lyons et al. (2013) oder auch Wardman und Lyons (2016) an, die den Wert von Reisezeit insbesondere im Sinne einer Nutzbarmachung durch die Reisenden hervorheben. Im Hinblick auf die Aktivitäten von Pendlern zwischen Köln und Bonn haben sich zudem ähnliche Muster gezeigt wie bereits in den Untersuchungen von Gamberini et al. (2012) in London und auch Camacho et al. (2017) in Australien.

Abschließend kann festgehalten werden, dass die im deutschsprachigen Raum bislang meist einseitige Betrachtung des Pendelns als ,Problem“ durch die Konzentration auf die damit verbundenen Herausforderungen und Einschränkungen in den Medien und der Wissenschaft der Thematik nicht vollständig gerecht wird. Der Arbeitsweg wird auf verschiedene Weisen genutzt und wahrgenommen - beruflich zwischen zwei Städten zu pendeln, wird längst nicht immer als Belastung empfunden, sondern kann auch als ein Mehrwert interpretiert werden.

Datenverfügbarkeit. Für diesen Artikel wurden qualitative Interviews mit Pendlern geführt. Den Gesprächspartner wurde Anonymität zugesagt. Die Transkripte sind deshalb nicht einsehbar.

Interessenkonflikt. Die Autoren erklären, dass kein Interessenkonflikt besteht. 
Danksagung. Für wertvolle Hinweise und Anregungen bedanken wir uns bei den beiden Gutachtern, mit denen wir allerdings leider nicht über unser Manuskript sprechen konnten. Stattdessen war uns Moritz Ochsmann ein wertvoller Gesprächspartner, dem wir ebenso für seine Mitwirkung an der Überarbeitung des Manuskripts danken.

\section{Edited by: Benedikt Korf}

Reviewed by: two anonymous referees

\section{Literatur}

Abraham, M. und Schönholzer, T.: Warum Pendeln nicht alle Probleme löst: Präferenzen für unterschiedliche Mobilitätsformen in „dual career“-Partnerschaften, Z. Familienforsch., 24, 229-246, 2012.

Bemmer, A.: Pendlerleben als Problem, Der Tagesspiegel, online aufrufbar: http://www.tagesspiegel.de/politik/ arbeitswelt-pendlerleben-als-problem/19608992.html, letzter Zugriff: 29.05.2017.

Boltze, M., Specht, G., Friedrich, D. und Figur, A.: Grundlagen für die Beeinflussung des individuellen Verkehrsmittelwahlverhaltens durch Direktmarketing, Selbstverlag der TU Darmstadt, Darmstadt, 2002.

BBSR (Bundesinstitut für Bau-, Stadt- und Raumforschung) (Hrsg.): Verkehrsbild Deutschland. Pendlerströme. Quo navigant?, BBSR-Analysen KOMPAKT 15, Bonn, 2015.

BBSR (Bundesinstitut für Bau-, Stadt- und Raumforschung) (Hrsg.): Immer mehr Menschen pendeln zur Arbeit, online aufrufbar: http://www.bbsr.bund.de/BBSR/DE/Home/Topthemen/ 2017-pendeln.html, letzter Zugriff: 25.05.2017.

BMVI (Bundesministerium für Verkehr und digitale Infrastruktur) (Hrsg.): Verkehr in Zahlen 2016/2017, Hamburg, 2016.

Bundeszentrale für politische Bildung: Erwerbstätigkeit von Frauen, online aufrufbar: www.bpb.de/system/files/pdf/O4NQPA.pdf (letzter Zugriff: 28.04.2017), 2010.

Camacho, T., Foth, M., Rakotonirainy, A., und Rittenbruch, M.: Understanding urban rail invehicle activities: An activity theory approach, Transport. Res. F-Traf., 46, 70-86, 2017.

Eckey, H.-F., Kosfeld, R. und Türck, M.: Pendelbereitschaft von Arbeitnehmern in Deutschland, Raumforschung und Raumordnung $65,5-15,2007$.

Flick, U.: Qualitative Sozialforschung. Eine Einführung, Rowohlt Taschenbuch Verlag, Reinbek bei Hamburg, 2011.

Gamberini, L., Spagnolli, A., Miotto, A., Ferrari, E., Corradi, N. und Furlan, S.: Passengers' activities during short trips on the London Underground, Transportation 40, 251-268, 2013.

Gottschall, K. und Voß, G. G. (Hrsg.): Entgrenzung von Arbeit und Leben. Zum Wandel von Erwerbstätigkeit und Privatsphäre im Alltag, München, 2. Auflage, 2005.

Information und Technik Nordrhein-Westfalen (IT.NRW): Ausgewählte Daten für Bonn und Köln [E-Mail], (Persönliche Mitteilung vom 06.04.2017), 2017.

Jain, J. und Lyons, G.: The gift of travel time, J. Transp. Geogr., 16, 81-89, 2008.

Jurczyk, K., Schier, M., Szymenderski, P., Lange, A. und Voß, G. G.: Entgrenzte Arbeit - Entgrenzte Familie. Grenzmanagement im Alltag als neue Herausforderung, Forschung aus der HansBöckler-Stiftung 100, Berlin, 2009.
Kahneman, D., Krueger, A.-B., Schkade, D. A., Schwarz, N. und Stone, A. A.: A survey method for characterizing daily life experience: the day reconstruction method, Science 306, 1776-1780, 2004.

Kemen, J.: Mobilität und Gesundheit. Einfluss der Verkehrsmittelnutzung auf die Gesundheit Berufstätiger, Springer Spektrum, Berlin, 2016.

Kluge, S.: Empirisch begründete Typenbildung: Zur Konstruktion von Typen und Typologien in der qualitativen Sozialforschung, Springer Verlag, Wiesbaden, 1999.

Kratzer, N. und Sauter, D.: Entgrenzung von Arbeit. Konzept, Thesen. Befunde, in: Entgrenzung von Arbeit und Leben. Zum Wandel von Erwerbstätigkeit und Privatsphäre im Alltag, Herausgeber: Gottschall, K. und Voß, G. G., München, 2. Auflage, 2005.

Kümmerling, A., Jansen, A. und Lehndorf, S.: Immer mehr Frauen sind erwerbstätig - aber mit kürzeren Wochenarbeitszeiten. Aktuelle Forschungsergebnisse aus dem Institut Arbeit und Qualifikation, IAQ-Report 4, online aufrufbar: https://www.boeckler. de/pdf_fof/96780.pdf (letzter Zugriff: 27.04.2017), 2008.

Läpple, D., Mückenberger, U. und J. Oßenbrügge (Hrsg.): Zeiten und Räume der Stadt. Theorie und Praxis, Opladen, 2010.

Lyons, G. und Urry, J.: Travel time use in the information age, Transportation Res. A-Pol., 39, 257-276, 2005.

Lyons, G., Jain, J., Susilo, Y. und Atkins, S.: Comparing Rail Passengers' Travel Time Use in Great Britain Between 2004 and 2010, Mobilities, 8, 560-579, 2013.

Mattissek, A., Pfaffenbach, C. und Reuber, P.: Methoden der empirischen Sozialforschung, Westermann, Braunschweig, 2013.

Matzig, G.: Beam mich aufs Land Scotty, Süddeutsche Zeitung [01.08.2017], 2017.

Menzl, M.: Leben in Suburbia. Raumstrukturen und Alltagspraktiken am Rande von Hamburg, Frankfurt, 2007.

Müller, B.: Land der Pendler, Süddeutsche Zeitung [02.04.2017], 2017.

Oostendorp, R.: Wohnstandortentscheidungen von Doppelverdienerhaushalten. Zum Zusammenwirken von Wohnen und Arbeiten in der polyzentrischen Stadtregion Köln/Bonn, Schriften des Arbeitskreises Stadtzukünfte 16, LIT-Verlag, Münster, 2016.

Ott, E. und Gerlinger, T.: Die Pendlergesellschaft. Zur Problematik der fortschreitenden Trennung von Wohn- und Arbeitsort, Bund Verlag, Köln, 1992.

Pfaff, S.: Pendeln oder Umziehen? Mobilitätsentscheidungen in Deutschland zwischen 2000 und 2009, Z. Soziol., 41, 458-477, 2012.

Pfaff, S.: Pendelentfernung, Lebenszufriedenheit und Entlohnung. Eine Längsschnittuntersuchung mit den Daten des SOEP von 1998 bis 2009, Z. Soziol., 43, 113-130, 2014.

Pohl, T.: Entgrenzte Stadt. Räumliche Fragmentierung und zeitliche Flexibilisierung in der Spätmoderne, Transcript Verlag, Bielefeld, 2009.

Poppitz, A.: Beruflich Bahnfahren: Aneignung des arbeitsbedingten Bahnalltags bei Pendlern und Geschäftsreisenden, Rainer Hampp Verlag, München, Mering, 2009.

Rau, R.: Zur Wechselwirkung von Arbeit, Beanspruchung und Erholung, in: Gesundheitsförderung und Gesundheitsmanagement in der Arbeitswelt. Ein Handbuch, Herausgeber: Bamberg, E., Ducki, A. und Metz, A.-M., Göttingen, Hogrefe Verlag, 83-106, 2011. 
Redmond, L. S. und Mokhtarian, P. L.: The positive utility of the commute: modeling ideal commute time and relative desired commute amount, Transportation 28, 179-205, 2001.

Reuber, P.: Heimat in der Großstadt. Eine sozialgeographische Studie zu Raumbezug und Entstehung von Ortsbindung am Beispiel Kölns und seiner Stadtviertel, Kölner Geographische Arbeiten 58, Köln, 1993.

Ruppenthal, S. und Lück, D.: Jeder fünfte Erwerbstätige ist aus beruflichen Gründen mobil. Berufsbedingte räumliche Mobilität im Vergleich, Informationsdienst Soziale Indikatoren 42, 1-5, 2009.

Schneider, N. F., Limmer, R. und Ruckdeschel, K.: Berufsmobilität und Lebensform. Sind berufliche Mobilitätserfordernisse in Zeiten der Globalisierung noch mit der Familie vereinbar?, Schriftenreihe des Bundesministeriums für Familie, Senioren, Frauen und Jugend 208, Stuttgart, 2002a.

Schneider, N. F., Limmer, R. und Ruckdeschel, K.: Mobil, flexibel, gebunden. Familie und Beruf in der mobilen Gesellschaft, Campus-Verlag, Frankfurt, 2002b.
Statistisches Bundesamt (Hrsg.): Berufspendler: Infrastruktur wichtiger als Benzinpreis, STATmagazin: Arbeitsmarkt 5, Wiesbaden, 2014.

Statistisches Bundesamt (Hrsg.): Statistisches Jahrbuch. Deutschland und Internationales, Wiesbaden, 2016.

Tagesschau.de: Zahl der Pendler erreicht Rekord, online aufrufbar: https://meta.tagesschau.de/id/121943/ zahl-der-pendler-erreicht-rekord, letzter Zugriff: 06.08.2017.

Wardman, M. und Lyons, G.: The digital revolution and worthwhile use of travel time: implications for appraisal and forecasting, Transportation, 43, 507-530, 2016.

Wörmer, S.: Berufliche Mobilität im Alltag. Praktiken und Formen alltäglicher Lebensführung, Schriften des Arbeitskreises Stadtzukünfte 17, LIT-Verlag, Münster, 2016. 